\title{
Tuberculosis among older adults in Zambia: burden and characteristics among a neglected group
}

\author{
Jenna Coffman ${ }^{1 *}$, Pascalina Chanda-Kapata ${ }^{2}$, Ben J. Marais ${ }^{3,4}$, Nathan Kapata $^{5}$, Alimuddin Zumla ${ }^{6}$ and Joel Negin ${ }^{1,3}$ (D)
}

\begin{abstract}
Background: The 2010 Global Burden of Disease estimates show that $57 \%$ of all TB deaths globally occurred among adults older than 50 years of age. Few studies document the TB burden among older adults in Southern Africa. We focused on adults older than 55 years to assess the relative TB burden and associated demographic factors.

Methods: A cross sectional nationally representative TB prevalence survey conducted of Zambian residents aged 15 years and above from 66 clusters across all the 10 provinces of Zambia. Evaluation included testing for TB as well as an in-depth questionnaire. We compared survey data for those aged 55 and older to those aged 15-54 years. Survey results were also compared with 2013 routinely collected programmatic notification data to generate future hypotheses regarding active and passive case finding.

Results: Among older adults with TB, 30/ 54 (55.6\%) were male, 3/27 (11.1\%) were HIV infected and 35/54 (64.8\%) lived in rural areas. TB prevalence was higher in those aged $\geq 55(0.7 \%)$ than in the $15-54$ age group (0.5\%). Males had higher rates of TB across both age groups with $0.7 \%(15-54)$ and $1.0 \%(\geq 55)$ compared with females $0.4 \%(15-54)$ and $0.6 \%$ ( 255$)$. In rural areas, the prevalence of TB was significantly higher among older than younger adults ( $0.7 \%$ vs $0.3 \%)$, while the HIV infection rate was among TB patients was lower (11.1\% vs 30.8\%). The prevalence survey detected TB in 54/7484 (0.7\%) of older adults compared to 3619/723,000 (0.5\%) reported in 2013 programmatic data.
\end{abstract}

Conclusion: High TB rates among older adults in TB endemic areas justify consideration of active TB case finding and prevention strategies.

Keywords: Tuberculosis, Older adults, Zambia

\section{Background}

Global efforts to reduce mortality from tuberculosis (TB) has shown solid progress with mortality rates decreasing over the past decade [1]. Programs on prevention, screening and treatment have contributed to these declines among certain groups such as those vulnerable to developing TB such as children [2], those living with HIV [3], and people with diabetes [4]. The World Health Organization estimates that the global case detection rate reached $64 \%$ in 2013 and that treatment success rates were above $85 \%$ [1]. However, despite the progress achieved and increased attention on certain groups, there has been ongoing neglect of older

\footnotetext{
* Correspondence: jennacoffman@live.com.au

'School of Public Health, University of Sydney, Sydney, Australia

Full list of author information is available at the end of the article
}

adults - those aged 50 years and older, especially in low and middle-income countries [5].

Older adults' vulnerability to TB has long been recognised [6]. Data from the 2015 Global Burden of Disease studies show that $62 \%$ of all tuberculosis deaths globally occurred among adults older than $50[7,8]$. The World Health Organization acknowledges that some regions have seen a progressive increase in the prevalence of $\mathrm{TB}$ with age [1] which has been confirmed in studies from countries such as China [9, 10], India [11, 12], and the Americas [13, 14]. But in Southern Africa, which remains the region of the world most affected by tuberculosis with the highest incidence and prevalence of the disease [8], the age distribution of the disease is often poorly defined. The TB challenge in Southern Africa is 
exacerbated by the HIV epidemic - which itself is ageing with the success of anti-retroviral treatment $[15,16]$.

Few studies exist on the TB burden among older adults in Southern Africa. Karstaedt and Bolhaar found a high burden of TB among the elderly in Soweto and that $50 \%$ of the older adults with TB had extrapulmonary tuberculosis [17]. Blaser and colleagues' agestructured transmission model for Cape Town found that simulated TB notification rates gradually increased from the age of 30 years [18].

Understanding the burden of $\mathrm{TB}$ among older adults is important due to specific features of the disease and its diagnosis among this age group. Older adults are more vulnerable to developing TB disease and are more likely to develop atypical forms of TB such as tuberculous meningitis, and sputum smear-negative pulmonary involvement which are often harder to diagnose and treat [19-21]. Diagnosis is also more challenging as common symptoms used for diagnosis are less prominent in older populations [21] and the common Mantoux skin test tends to produce false negative results in older patients. [21-24] Furthermore, vulnerability among older adults is increased due to increased rates of compromised immune systems due to co-morbidity such as diabetes [25] and cancers [5].

We therefore analysed the 2013-14 Zambia TB prevalence survey data focusing specifically on older adults aged 55 years and above, in order to better understand TB burden and associated demographic factors in this population in a high-burden country. In addition, we sought to compare the survey data to routine national notification data. Zambian TB incidence has been estimated as of 2015 at 205.9 per 100,000 population with 3573 deaths (22.0 per 100,000 population)by the Global Burden of Disease study [8].

\section{Methods}

The 2013-14 Zambia TB prevalence survey was a cross sectional survey of the prevalence of $\mathrm{TB}$ among residents aged 15 years and above from 66 clusters across all 10 provinces of Zambia. In the initial census, 98,458 participants were identified. Symptom and chest $\mathrm{x}$-ray screening was offered to all survey participants after obtaining consent. There was a $84.1 \%$ participation rate from the participants originally identified. Those who reported any relevant symptoms and/or were found to have an abnormal chest $\mathrm{x}$-ray underwent an in-depth questionnaire. In addition, they were requested to submit a spot and morning sputum sample for MTB identification which was identified by either liquid culture (using MGIT BACTEC $960^{\mathrm{mm}}$ ). Indeterminate sputum smear samples where there was no conclusive culture results were subjected to Xpert MTB Rif. HIV counselling and testing was offered to all survey participants. The detailed national TB prevalence survey procedures and HIV testing procedures are reported elsewhere [26, 27].

In this analysis, survey data for those aged 55 and older was extracted from the main survey database. The variables extracted included participant age, residence, employment status, education level and socio-economic status. The prevalence of $\mathrm{TB}$ and socio demographic characteristics among those aged 55 years and above were compared with those aged 15-54 years. Odds ratios $(\mathrm{OR})$ and confidence intervals were produced using logistic regression, accounting for clustered sampling with 66 primary sampling units (PSU). Socioeconomic status was determined by deriving wealth indices using data collected on household assets, housing characteristics and access to utilities. This was achieved using the standard asset ownership questionnaire used in the Zambia Demographic Health Surveys,

The survey data were then compared with 2013 routinely collected data to ascertain whether there was a difference in rates of TB obtained between the two forms of data collection. An implied prevalence based on total population disaggregated by age and province was calculated. The 2013 routinely collected data was available from Ministry of Health national TB notification reports.

Data were analysed with SPSS version 22 software (IBM Corp. Released 2013. IBM SPSS Statistics for Mac, Version 22. AMonk, NY: IBM Corp.) and Stata Statistical Software: Release 12 (StataCorp, 2011). Overall TB prevalence rates were calculated; additional subgroup analyses were done to calculate specific prevalence rates by age, sex, geographical location, employment status, education level, and HIV status. Health seeking behaviour was analysed to assess access to health facilities.

The Zambian National TB prevalence survey study protocol was cleared by the University of Zambia Biomedical Research Ethics Committee (UNZABREC) No: 020-08-12.

\section{Results}

Data for the Zambia national tuberculosis prevalence survey was collected between August 2013 and July 2014. The study identified 98,458 individuals in 66 clusters. Of these, 54,830 (55.7\%) were residents and were aged 15 years and older and were therefore eligible to participate. Of these, 46,099 (84.1\%) underwent symptom screening, while 45,633 (99\%) were screened by chest $x$-ray (CXR) at field level. From these, 4453 had positive symptom screenings while $3758(8.2 \%)$ had an abnormal CXR. The mean age of the participants was 36.1. Of all respondents, $16.2 \%$ were aged 55 and older.

Among participants, the overall prevalence of TB was higher in those aged 55 and older (0.7\%) than in the 1554 age group (0.5\%) (Table 1 ). Males had significantly higher rates of $\mathrm{TB}$ across both age groups with $0.7 \%$ 
Table 1 Number of people screened for tuberculosis in the Zambian national tuberculosis prevalence survey 2013-14, proportion of people diagnosed with tuberculosis and association of age group (15-54 years and 55 years and above) with demographic characteristics

\begin{tabular}{|c|c|c|c|c|c|c|}
\hline \multicolumn{2}{|l|}{ Demographic characteristic } & \multicolumn{2}{|l|}{$15-54$} & \multicolumn{2}{|l|}{$55+$} & \multirow{2}{*}{$\begin{array}{l}\text { Odds Ratios } \\
\text { (95\% Confidence intervals }\end{array}$} \\
\hline & & Population & Number of Cases & Population & Number of Cases & \\
\hline Overall & & 38,619 & $211(0.5 \%)$ & 7484 & $54(0.7 \%)$ & $1.32(0.95-1.86)$ \\
\hline \multirow[t]{2}{*}{ Sex } & Male & 16,315 & $122(0.7 \%)$ & 3145 & $30(1.0 \%)$ & $1.28(0.80-2.04)$ \\
\hline & Female & 22,304 & $89(0.4 \%)$ & 4339 & $24(0.6 \%)$ & $1.39(0.89-2.17)$ \\
\hline \multirow[t]{2}{*}{ Location } & Urban & 13,783 & $126(0.9 \%)$ & 2274 & 19 (0.8\%) & $0.92(0.58-1.44)$ \\
\hline & Rural & 24,836 & $85(0.3 \%)$ & 5210 & $35(0.7 \%)$ & $1.97(1.35-2.89)$ \\
\hline \multirow[t]{10}{*}{ Province } & Central & 2403 & $12(0.5 \%)$ & 503 & $1(0.2 \%)$ & $0.40(0.08-1.97)$ \\
\hline & Copperbelt & 6608 & $74(0.1 \%)$ & 1628 & 19 (1.2\%) & $1.04(0.51-2.14)$ \\
\hline & Eastern & 5990 & $8(0.1 \%)$ & 1167 & $4(0.3 \%)$ & $2.57(1.86-3.55)$ \\
\hline & Luapula & 3013 & $7(0.2 \%)$ & 646 & $2(0.3 \%)$ & $1.33(0.31-5.69)$ \\
\hline & Lusaka & 7414 & $63(0.9 \%)$ & 1128 & $8(0.7 \%)$ & $0.83(0.49-1.42)$ \\
\hline & Muchinga & 1052 & $2(0.2 \%)$ & 199 & $0(0.0 \%)$ & NA \\
\hline & Northern & 3296 & $17(0.5 \%)$ & 594 & $10(1.7 \%)$ & $3.31(1.81-6.05)$ \\
\hline & North Western & 2310 & $9(0.4 \%)$ & 530 & $4(0.8 \%)$ & $1.94(1.24-3.06)$ \\
\hline & Southern & 5089 & $11(0.2 \%)$ & 594 & $4(0.7 \%)$ & $3.12(0.74-13.19)$ \\
\hline & Western & 1444 & $8(0.6 \%)$ & 495 & $2(0.4 \%)$ & $0.73(0.29-1.82)$ \\
\hline \multirow[t]{2}{*}{ Employment Status $^{\text {a }}$} & Unemployed & 17,951 & $102(0.6 \%)$ & 2779 & $22(0.8 \%)$ & $1.40(0.84-2.33)$ \\
\hline & Employed & 20,405 & $109(0.5 \%)$ & 4494 & $29(0.6 \%)$ & $1.21(0.82-1.79)$ \\
\hline \multirow[t]{4}{*}{ Highest Level of Education } & No Schooling & 2172 & $8(0.4 \%)$ & 2107 & $18(0.9 \%)$ & $2.33(0.99-5.49)$ \\
\hline & Primary & 16,759 & $98(0.6 \%)$ & 3916 & $24(0.6 \%)$ & $1.05(0.61-1.79)$ \\
\hline & Secondary & 18,081 & $100(0.6 \%)$ & 1243 & $9(0.7 \%)$ & $1.32(0.68-2.55)$ \\
\hline & Tertiary & 1576 & $5(0.3 \%)$ & 218 & $3(1.4 \%)$ & $4.35(0.73-25.85)$ \\
\hline \multirow[t]{5}{*}{ Socioeconomic Status ${ }^{\text {b }}$} & Lowest & 7374 & $31(0.4 \%)$ & 1474 & $4(0.3 \%)$ & $0.65(0.26-1.63)$ \\
\hline & Lower & 6765 & $29(0.4 \%)$ & 1366 & $8(0.6 \%)$ & $1.37(0.69-2.73)$ \\
\hline & Middle & 7099 & $51(0.7 \%)$ & 1370 & $14(1.0 \%)$ & $1.43(0.64-3.18)$ \\
\hline & Higher & 5914 & $39(0.7 \%)$ & 1123 & $14(1.2 \%)$ & $1.90(0.93-3.91)$ \\
\hline & Highest & 4991 & $25(0.5 \%)$ & 849 & $3(0.4 \%)$ & $0.70(0.21-2.40)$ \\
\hline
\end{tabular}

Denominator is the total number of eligible individuals who took part in the study and fit into the relevant category being assessed ${ }^{a}$ Employed includes occasional seasonal workers, government employees, private sector employees, self-employed, working on own land. Unemployed includes unemployed, students and housewife/homemakers

${ }^{b}$ Socioeconomic status was determined by deriving wealth indices using data collected on household assets, housing characteristics and access to utilities. This was achieved using the standard asset ownership questionnaire used in the Zambia Demographic Health Surveys

(15-54) and 1.0\% (55+) compared with females $(0.4 \%$ (15-54) and $0.5 \%(55+)$ ) (Table 1). In rural areas, the prevalence of TB was significantly higher among older adults than younger adults. Prevalence was highest in the Copperbelt province for both younger and older adults. The unemployed had a higher prevalence of TB across both age groups with $0.6 \%(15-54)$ and $0.8 \%$ (55 +) compared with the employed $(0.5 \%(15-54)$ and $0.6 \%$ $(55+))$. Tertiary educated older adults had the highest prevalence of TB (1.4\%) across both age groups. TB prevalence across socioeconomic quintiles did show a trend with the highest rates of TB seen in the 3rd and 4th quintiles across both groups with slightly higher prevalence among older adults.
Of older adults diagnosed with $\mathrm{TB}$ the mean age was 66 (Table 2). Of those older adults living with TB, 55.6\% were male. Compared to those aged 15-54, older adults living with $\mathrm{TB}$ were much more likely to be living in rural areas. Of older adults that were TB positive, 11.1\% were also HIV positive.

We compared the survey data with 2013 routinely collected data to ascertain whether there was a difference between the outcomes from the two forms of data collection (Table 3). Overall rate of TB among those aged 15-54 was the same between the survey and routine collection but for older adults (55+), survey prevalence was higher $(0.7 \%)$ compared to the implied prevalence from routine collection $(0.5 \%)$. This was 
Table $\mathbf{2}$ Characteristics of tuberculosis positive people identified in the Zambian national tuberculosis prevalence survey, 2013-14, by age group (15-54 years and 55 years and above)

\begin{tabular}{|c|c|c|c|}
\hline \multicolumn{2}{|l|}{ Characteristics } & \multirow{2}{*}{$\begin{array}{l}\text { Younger } \\
(15-54 \mathrm{yrs}) \\
n=211\end{array}$} & \multirow{2}{*}{$\begin{array}{l}\text { Older } \\
(\geq 55 \text { yrs }) \\
n=54 \\
66\end{array}$} \\
\hline Mean age & & & \\
\hline Sex & Male & $122(57.8 \%)$ & $30(55.6 \%)$ \\
\hline \multirow[t]{2}{*}{ Location } & Urban & $126(59.7 \%)$ & $22(40.3 \%)$ \\
\hline & Rural & $74(35.2 \%)$ & $35(64.8 \%)$ \\
\hline \multirow[t]{4}{*}{ Education } & No schooling & $8(3.8 \%)$ & $18(33.3 \%)$ \\
\hline & Primary & $98(46.5 \%)$ & $24(44.4 \%)$ \\
\hline & Secondary & $100(47.4 \%)$ & $9(16.7 \%)$ \\
\hline & Tertiary & $5(2.4 \%)$ & $3(5.6 \%)$ \\
\hline \multirow[t]{6}{*}{ Socioeconomic status } & & $n=175$ & $n=43$ \\
\hline & Lowest & $31(17.7 \%)$ & $4(9.3 \%)$ \\
\hline & Lower & $29(16.6 \%)$ & $8(18.6 \%)$ \\
\hline & Middle & $51(29.1 \%)$ & $14(32.6 \%)$ \\
\hline & Higher & $39(22.3 \%)$ & $14(32.6 \%)$ \\
\hline & Highest & $25(14.3 \%)$ & $3(7 \%)$ \\
\hline \multirow[t]{4}{*}{ Number in Household } & & $n=184$ & $n=53$ \\
\hline & $1-4$ & $45(24.5 \%)$ & $13(24.5 \%)$ \\
\hline & $5-8$ & $101(54.9 \%)$ & $28(52.8 \%)$ \\
\hline & $9+-12$ & $38(20.7 \%)$ & $12(22.7 \%)$ \\
\hline \multirow[t]{2}{*}{ Family member treated for TB } & & $n=210$ & $n=51$ \\
\hline & Yes & $38(18.1 \%)$ & $3(5.9 \%)$ \\
\hline \multirow{2}{*}{$\begin{array}{l}\text { HIV-infected (among those } \\
\text { with TB) }\end{array}$} & & $(n=107)$ & $(n=27)$ \\
\hline & & $33(30.8 \%)$ & $3(11.1 \%)$ \\
\hline \multirow{4}{*}{$\begin{array}{l}\text { Transport used to visit } \\
\text { nearest facility }\end{array}$} & & $n=211$ & $\mathrm{n}=54$ \\
\hline & Walk & $188(89.1 \%)$ & $42(77.8 \%)$ \\
\hline & Bicycle & $8(3.8 \%)$ & $6(11.1 \%)$ \\
\hline & Other & $15(7.1 \%)$ & $0(0 \%)$ \\
\hline \multirow[t]{3}{*}{ Travel time to nearest facility } & $<1 \mathrm{~h}$ & $173(82 \%)$ & $38(70.7 \%)$ \\
\hline & 1 to $3 \mathrm{~h}$ & $34(16.1 \%)$ & $15(27.8 \%)$ \\
\hline & $>3 \mathrm{~h}$ & $4(1.9 \%)$ & $1(1.9 \%)$ \\
\hline
\end{tabular}

particularly pronounced for older males where survey prevalence was $1.0 \%$ compared to $0.6 \%$ from routine data. Data from Copperbelt Province found double the TB prevalence among older adults in the survey than found in routine data collection and Northern Province - the largest and most remote province - found a much higher rate of TB through the survey as through routine data.

\section{Discussion}

Analysis of the Zambia TB prevalence survey found high rates of TB among those aged 55 years and older compared to younger age groups. This difference was consistent among men and women and was particularly pronounced among older adults living in rural areas. Older adults in higher socioeconomic status bands had higher TB prevalence than younger adults in those bands. HIV prevalence was lower among older adults living with $\mathrm{TB}$ than among younger adults living with $\mathrm{TB}$ suggesting that co-morbidity is less of a driver of $\mathrm{TB}$ among the older population. The rates of TB were found to be higher among older adults in the survey than what was found from notification data that was collected through routine services, suggesting that some older adults are being missed by currently available health services.

The fact that the high burden of $\mathrm{TB}$ among older adults is not as associated with HIV infection is notable. In 2012, a national tuberculosis survey done in Tanzania demonstrated an epidemic shift from young HIVinfected to older HIV-uninfected people [28]. The highest TB prevalence rate found in that survey was among those aged 45 years and older in whom HIV prevalence rates were generally lower. Though HIV prevalence among older adults is increasing [16, 29], there is need to better understand the determinants and drivers of the TB burden among the older population.

The suggested burden of undiagnosed TB among older adults has been found in other LMIC settings as well. In urban Cambodia, a community-based active case finding project found that $26 \%$ of new cases found were older than 65 which was double the proportion found through passive case finding $[30,31]$. Together, this suggests that the current emphasis on HIV and TB co-morbidity is not sufficient to address the challenges of this underdiagnosed pool of older HIV-uninfected older adults who may have particular challenges with regard to health access due to poverty, rural living, mobility difficulties [32] and ageist stigma [33]. There is currently limited evidence regarding successful interventions for the improvement of diagnosis of TB among older adults in LMICs [34].

Relatively high rates of TB among older adults challenge the current $\mathrm{TB}$ response and require new data and thinking. Li and colleagues' recent scoping review [34] identified a number of causal pathways to improve TB prevention and services among older people. While the review focused on articles from high-income countries [34], some of the interventions might be relevant in lowand middle-income settings. Screening of latent TB infection for example reduced the risk of reactivation $[35,36]$ and the review authors promoted active systematic screening for high-risk groups to reduce the delay in diagnosis and containment. Improving the health of older adults living with $\mathrm{TB}$ is important not only for their health but, additionally, older carers with TB pose a risk to the young children in their care. Therefore, programs have the potential to be framed as a lifespan intervention. 
Table 3 Tuberculosis prevalence among older adults (aged 55+) based on survey results compared to routinely collected programmatic data

\begin{tabular}{|c|c|c|c|c|}
\hline & & & Survey & Routine data collection \\
\hline \multirow[t]{2}{*}{ Overall prevalence } & & $15-54$ & $0.5 \%(38,816)$ & $0.5 \%(6,263,000)$ \\
\hline & & $55+$ & $0.7 \%(7512)$ & $0.5 \%(743,000)$ \\
\hline \multirow[t]{4}{*}{ Sex } & Male & $15-54$ & $0.7 \%(16,415)$ & $0.6 \%(3,148,000)$ \\
\hline & & $55+$ & $1.0 \%(3156)$ & $0.6 \%(349,000)$ \\
\hline & Female & $15-54$ & $0.4 \%(22,401)$ & $0.4 \%(3,128,000)$ \\
\hline & & $55+$ & $0.6 \%(4356)$ & $0.4 \%(393,000)$ \\
\hline \multirow[t]{18}{*}{ Provinces } & Central & $15-54$ & $0.5 \%(2471)$ & $0.3 \%(618,688)$ \\
\hline & & $55+$ & $0.2 \%(51)$ & $0.3 \%(70,490)$ \\
\hline & Copperbelt & $15-54$ & $1.1 \%(6608)$ & $0.7 \%(955,808)$ \\
\hline & & $55+$ & $1.2 \%(1628)$ & $0.6 \%(108,899)$ \\
\hline & Eastern & $15-54$ & $0.1 \%(5990)$ & $0.2 \%(833,373)$ \\
\hline & & $55+$ & $0.3 \%(1167)$ & $0.3 \%(94,950)$ \\
\hline & Luapula & $15-54$ & $0.2 \%(3081)$ & $0.3 \%(467,980)$ \\
\hline & & $55+$ & $0.3 \%(660)$ & $0.5 \%(53,319)$ \\
\hline & Lusaka & $15-54$ & $0.9 \%(7414)$ & $1.1 \%(1,073,110)$ \\
\hline & & $55+$ & $0.7 \%(1128)$ & $0.9 \%(122,264)$ \\
\hline & Northern & $15-54$ & $0.5 \%(3302)$ & $0.1 \%(858,685)$ \\
\hline & & $55+$ & $1.7 \%(594)$ & $0.2 \%(97,834)$ \\
\hline & North Western & $15-54$ & $0.4 \%(2310)$ & $0.3 \%(344,753)$ \\
\hline & & $55+$ & $0.8 \%(530)$ & $0.3 \%(39,279)$ \\
\hline & Southern & $15-54$ & $0.2 \%(5107)$ & $0.3 \%(784,115)$ \\
\hline & & $55+$ & $0.7 \%(597)$ & $0.5 \%(89,338)$ \\
\hline & Western & $15-54$ & $0.6 \%(1444)$ & $0.5 \%(430,184)$ \\
\hline & & $55+$ & $0.4 \%(495)$ & $1.0 \%(49,013)$ \\
\hline
\end{tabular}

Population figures from 2010 Zambian census

\section{Limitations}

We acknowledge the limitations of the study and subsequent analysis. Diagnosis of TB among older adults is difficult [21]. Further, due to the cross-sectional study design, we were unable to separate the independent influences of age, socioeconomic status, geographical location, education level, and employment status on TB prevalence in this study. Some of the differences we found with regard to younger versus older adults may be attributable to such confounders; we were unable to include a regression analysis to further analyse the relationship of age, TB status and associated risk factors due to the small numbers of those with TB for the over 55 age group. Further analyses will do so now that this preliminary analysis has generated hypotheses. Additionally, we acknowledge that the category of $15-54$ is a large age group and there are likely substantial differences within that group - but given our focus on older adults specifically, we have not performed analyses within that younger age range.

We acknowledge that comparing the study data with the routinely collected data is not comparing the same measurements but have included for interest and to encourage further research. Additionally, we acknowledge that $16.2 \%$ of respondents being $55+$ is an over-representation of this age group compared with the Zambian population but this also means that the data for this group was more robust than it would have been otherwise.

\section{Conclusion}

The findings from this national survey suggest that the national tuberculosis program in Zambia and similar settings should prioritise active case finding among older adults. In addition, prevention activities should be specifically targeted to older adults where they live and socialise [37].

\section{Acknowledgements}

None.

\section{Funding}

The survey was co-funded by the Government of the Republic of Zambia (GRZ) through the Ministry of Health $(\mathrm{MoH})$ and the United States Government (USG) through United States Agency for International Development-through the TB CARE I Project/ Centres for Disease Control and Prevention (USAID)/CDC/TB

CARE I. No funding was received for this analysis. 


\section{Availability of data and materials}

Due to the presence of identifying patient information, an anonymized dataset is available upon request from the Principal Investigator (nkapata@gmail.com).

\section{Authors' contributions}

Conceived and designed the experiments: NK PCK AZ; Performed the experiments: NK PCK; Analyzed the data: JC JN; Wrote the first draft: JC JN; Reviewed the draft and provided input: JN NK PCK BM AZ; Provided input to the analysis: NK PCK BM; Edited and provided guidance to analytical approach: AZ BM NK. All authors read and approved the final manuscript.

\section{Ethics approval and consent to participate}

The Zambian National TB prevalence survey study protocol was cleared by the University of Zambia Biomedical Research Ethics Committee (UNZABREC) No: 020-08-12. Written informed consent was obtained from all individuals who agreed to participate in the survey. For minors, both the assent of the minor and the consent of the next of kin or caretakers or guardian was obtained in writing for each participant aged 15-17 years. The institutional review board (IRB) approved this consent procedure.

\section{Consent for publication}

Not applicable.

\section{Competing interests}

The authors declare that they have no competing interests.

\section{Publisher's Note}

Springer Nature remains neutral with regard to jurisdictional claims in published maps and institutional affiliations.

\section{Author details}

${ }^{1}$ School of Public Health, University of Sydney, Sydney, Australia. ${ }^{2}$ Department of Disease Surveillance, Control and Research, Ministry of Health, Lusaka, Zambia. ${ }^{3}$ Marie Bashir Institute for Infectious Diseases and Biosecurity, University of Sydney, Sydney, Australia. ${ }^{4}$ Children's Hospital at Westmead, University of Sydney, Sydney, Australia. ${ }^{5}$ National TB and Leprosy Control Program, Lusaka, Zambia. ${ }^{6}$ Division of Infection and Immunity, Department of Infection, University College London, London, UK.

\section{Received: 23 March 2017 Accepted: 6 October 2017}

Published online: 12 October 2017

\section{References}

1. World Health Organization. Global Tuberculosis Report 2016. Geneva: World Health Organization; 2016

2. Marais BJ, Schaaf HS, Graham SM. Child health and tuberculosis. Lancet Respir Med. 2014:2(4):254-6.

3. Raviglione MC, Narain JP, Kochi A. HIV-associated tuberculosis in developing countries: clinical features, diagnosis, and treatment. Bull World Health Organ. 1992;70(4):515-26

4. van Crevel R, Dockrell HM, Consortium TTANDEM. Understanding diabetes and tuberculosis. Lancet Diabetes Endocrinol. 2014;2(4):270-2.

5. Negin J, Abimbola S, Marais BJ. Tuberculosis among older adults-time to take notice. Int J Infect Dis. 2015:32:135-7.

6. Schaaf HS, Collins A, Bekker A, Davies PD. Tuberculosis at extremes of age. Respirology. 2010;15(5):747-63.

7. Institute for Health Metrics and Evaluation. Global Burden of Disease. 2014 http://www.healthdata.org/gbd.

8. Murray CJ, Ortblad KF, Guinovart C, et al. Global, regional, and national incidence and mortality for HIV, tuberculosis, and malaria during 1990-2013: a systematic analysis for the Global Burden of Disease Study 2013. Lancet. 2014;384(9947):1005-70.

9. Abuaku B, Tan H, Li X, Chen M, Huang X. Treatment default and death among tuberculosis patients in Hunan, China. Scand J Infect Dis. 2010;42(4): 281-7.

10. Xia Y, Jiang $S$. The 5 th Nationwide TB prevalence survey in China. In: Control CCfD, ed. Lille; 2011.

11. Ananthakrishnan R, Kumar K, Ganesh M, et al. The profile and treatment outcomes of the older (aged 60 years and above) tuberculosis patients in Tamilnadu, South India. PLoS One. 2013;8(7):e67288.
12. Velayutham BR, Nair D, Chandrasekaran V, et al. Profile and response to antituberculosis treatment among elderly tuberculosis patients treated under the TB control programme in South India. PLoS One. 2014;9(3):e88045.

13. Organization PAH. Tuberculosis in the Region of the Americas Regional Report 2011: Epidemiology, Control and Financing. Washington, DC: Pan American Health Organization; 2011.

14. Menezes AM, Hallal PC, Perez-Padilla R, et al. Tuberculosis and airflow obstruction: evidence from the PLATINO study in Latin America. Eur Respir J. 2007:30(6):1180-5.

15. Mills EJ, Barnighausen T, Negin J. HIV and aging-preparing for the challenges ahead. N Engl J Med. 2012;366(14):1270-3.

16. Negin J, Cumming RG. HIV infection in older adults in sub-Saharan Africa: extrapolating prevalence from existing data. Bull World Health Organ. 2010; 88(11):847-53

17. Karstaedt AS, Bolhaar M. Tuberculosis in older adults in Soweto, South Africa. Int J Tuberc Lung Dis. 2014;18(10):1220-2.

18. Blaser N, Zahnd C, Hermans S, et al. Tuberculosis in cape town: an agestructured transmission model. Epidemics. Mar 2016;14:54-61.

19. Yoshikawa TT. Tuberculosis in aging adults. J Am Geriatr Soc. 1992:40(2): 178-87.

20. Pratt RH, Winston CA, Kammerer JS, Armstrong LR. Tuberculosis in older adults in the United States, 1993-2008. J Am Geriatr Soc. 2011:59(5):851-7.

21. Perez-Guzman C, Vargas MH, Torres-Cruz A, Villarreal-Velarde H. Does aging modify pulmonary tuberculosis?: a meta-analytical review. Chest. 1999; 116(4):961-7.

22. Pottumarthy S, Morris AJ, Harrison AC, Wells VC. Evaluation of the tuberculin gamma interferon assay: potential to replace the Mantoux skin test. J Clin Microbiol. 1999;37(10):3229-32.

23. Kobashi Y, Mouri K, Yagi S, et al. Clinical utility of the QuantiFERON TB-2G test for elderly patients with active tuberculosis. Chest. 2008;133(5):1196-202.

24. Rajagopalan S. Tuberculosis and aging: a global health problem. Clin Infect Dis. 2001;33(7):1034-9

25. Riza AL, Pearson F, Ugarte-Gil C, et al. Clinical management of concurrent diabetes and tuberculosis and the implications for patient services. Lancet Diabetes Endocrinol. 2014:2(9):740-53.

26. Kapata N, Chanda-Kapata P, Ngosa W, et al. The prevalence of tuberculosis in Zambia: results from the first national TB prevalence survey, 2013-2014. PLoS One. 2016. Forthcoming.

27. Chanda-Kapata P, Ngosa W, Moraes AN, Maddox N, Kapata N. Decliners of provider-initiated HIV testing and counselling: characteristics of participants who refused HIV testing in a population survey in Zambia. Asian Pac J Trop Med. 2015:5(8):689-93.

28. Law I. TB prevalence surveys: global overview. World Health Organization Global Tuberculosis Programme: Progress and Lessons Learnt; 2013.

29. Negin J, Barnighausen T, Lundgren JD, Mills EJ. Aging with HIV in Africa: the challenges of living longer. Aids. 2012;26(Suppl 1):S1-5.

30. Lorent N, Choun K, Thai S, et al. Community-based active tuberculosis case finding in poor urban settlements of Phnom Penh, Cambodia: a feasible and effective strategy. PLoS One. 2014;9(3):e92754.

31. World Health Organization. The Kingdom of Cambodia: Joint Review of the National TB Programme 2012. Manila: World Health Organization western Pacific Regional Office; 2012

32. Mabuza EM, Poggenpoel M, Myburgh C. Perceived basic needs and resources for the elderly in the peri-urban and rural communities in the Hhohho region in Swaziland. Curationis. 2010:33(1):23-32.

33. Waweru LM, Kabiru EW, Mbithi JN. Some ES. Health status and health seeking behaviour of the elderly persons in Dagoretti division, Nairobi. East Afr Med J. Feb 2003;80(2):63-7

34. Li J, Chung PH, Leung CLK, Nishikiori N, Chan EYY, Yeoh EK. The strategic framework of tuberculosis control and prevention in the elderly: a scoping review towards End TB targets. Infect Dis Pov. 2017:6(1):70.

35. Huynh GH, Klein DJ, Chin DP, et al. Tuberculosis control strategies to reach the 2035 global targets in China: the role of changing demographics and reactivation disease BMC Med. 2015:13:88.

36. Marchand $\mathrm{R}$, Tousignant $\mathrm{P}$, Chang $\mathrm{H}$. Cost-effectiveness of screening compared to case-finding approaches to tuberculosis in long-term care facilities for the elderly. Int J Epidemiol. 1999;28(3):563-70.

37. Zenner D, Southern J, van Hest R, et al. Active case finding for tuberculosis among high-risk groups in low-incidence countries. Int J Tuberc Lung Dis. 2013;17(5):573-82. 